\title{
Cluster Ions Ejected from an Li-Mg Alloy Liquid Metal Ion Source: Observation of $\mathrm{Mg}_{2}^{2+}$ and $\mathbf{M g}_{3}^{2+}$
}

\author{
Yahachi Saito \\ Department of Electrical Engineering, Faculty of Engineering, Mie University, Tsu, Japan \\ Toshiyuki Ishida* and Tamotsu Noda ${ }^{\dagger}$ \\ Department of Electrical and Electronic Engineering, Toyohashi University of Technology, Toyohashi, Japan
}

\begin{abstract}
Ions ejected from a liquid metal ion source of an $\mathrm{Li}-\mathrm{Mg}(10$ atom \%) alloy have been investigated by using a magnetic mass analyzer. In addition to singly charged homonuclear $\mathrm{Li}_{n}^{+}(n \leq 9)$ and $\mathrm{Mg}_{n}^{+}(n \leq 4)$ and heteronuclear $\mathrm{Mg}_{m} \mathrm{Li}_{n}^{+}(m, n \leq 2)$ clusters, doubly charged diatomic and triatomic $\mathrm{Mg}$ clusters are observed. Discussion is focused on the observability and the formation mechanism of the doubly charged small $\mathrm{Mg}$ clusters. A postionization process is suggested for the formation of the doubly charged clusters. (J Am Soc Mass Spectrom 1991, 2, 76-80)
\end{abstract}

$\mathrm{T}$ HE study of properties of atom clusters has expanded rapidly in the last decade since the advent of new techniques for producing clusters in the gas phase [1]. Mass spectrometry has been one of the primary tools used to probe size-dependent cluster properties such as size distribution, ionization energy, and chemical reactivity. In the measurement of cluster abundances, local maxima or discontinuous variations (steps) at certain specific cluster sizes are frequently encountered, and these sizes are referred to as "magic numbers."

Clusters are normally ionized prior to detection. Depending on the ionization process, more than two electrons can be removed, leaving multiply charged clusters, which have been found to be observable only above a threshold size ("critical size") [2]. The absence of doubly charged clusters below the critical number $n_{c}$ of constituents is explained by the explosion of the charged cluster caused by the repulsive Coulomb forces between the two holes.

Experimental and theoretical studies on the observability of multiply charged clusters were reviewed by Echt [3]. Tománek et al. [4] compared the binding energy $E_{\mathrm{B}}$ of a surface atom in the metallic cluster to the Coulomb energy $E_{C}$ under assumptions that the cluster is a spherical drop and that the two charges are at opposite sides of the drop. The critical numbers thus obtained, which correspond to critical sizes for spherical clusters, are in agreement with previous

*Present address: Japan Steel Works Ltd., Yotukaido 284, Japan. ${ }^{+}$Present address: Toyohashi Junior College, Toyohashi 440, Japan. Address reprint requests to Yahachi Saito, Department of Electrical Engineering, Faculty of Engineering, Mie University, Tsu 514, Japan. experimental observations for metallic clusters produced by the gas-aggregation technique [2]. However, doubly charged metallic clusters far below this critical size have been observed, especially in feld evaporation experiments. Drachsel et al. [5] discussed the existence of doubly charged triatomic clusters $\mathrm{M}_{3}^{2+}$ by assuming a linear chain structure with the two charges localized at the end atoms. A comparison with experimental data leads to a rough empirical criterion for stable $\mathrm{M}_{3}^{2+}, E_{\mathrm{C}}<1.4 E_{\mathrm{B}}$. Field emission and related techniques (pulsed-laser stimulated field evaporation, liquid metal ion source) easily produce ions in highly charged states. The charge states are probably formed by electron tunneling from the evaporated (singly charged) ions back into the tip. This postionization [6] appears to be the main reason for the frequent occurrence of small multiply charged clusters, because the energy transfer into the ionized cluster is considerably smaller in electron tunneling than in electron or photon impact ionization.

We have produced cluster ions from a liquid metal ion source (LMIS) of an $\mathrm{Li}-\mathrm{Mg}$ alloy. The original purpose of this experiment was to produce heteronuclear clusters and then study the effect of the differing chemical nature of the constituent atoms (valency and atom size) on the stability of the heteronuclear clusters. The observed heteronuclear clusters were limited to only three species $\left(\mathrm{MgLi}^{+}, \mathrm{MgLi}_{2}^{+}, \mathrm{Mg}_{2} \mathrm{Li}^{+}\right)$, but unexpectedly small multiply charged homonuclear clusters, $\mathrm{Mg}_{2}^{2+}$ and $\mathrm{Mg}_{3}^{2+}$, were detected. We have to use an $\mathrm{Li}-\mathrm{Mg}$ alloy as source material because pure magnesium has vapor pressure too high to operate the LMIS, but in the present paper the observation of $\mathrm{Mg}_{2}^{2+}$ and $\mathrm{Mg}_{3}^{2+}$ is the main interest. 


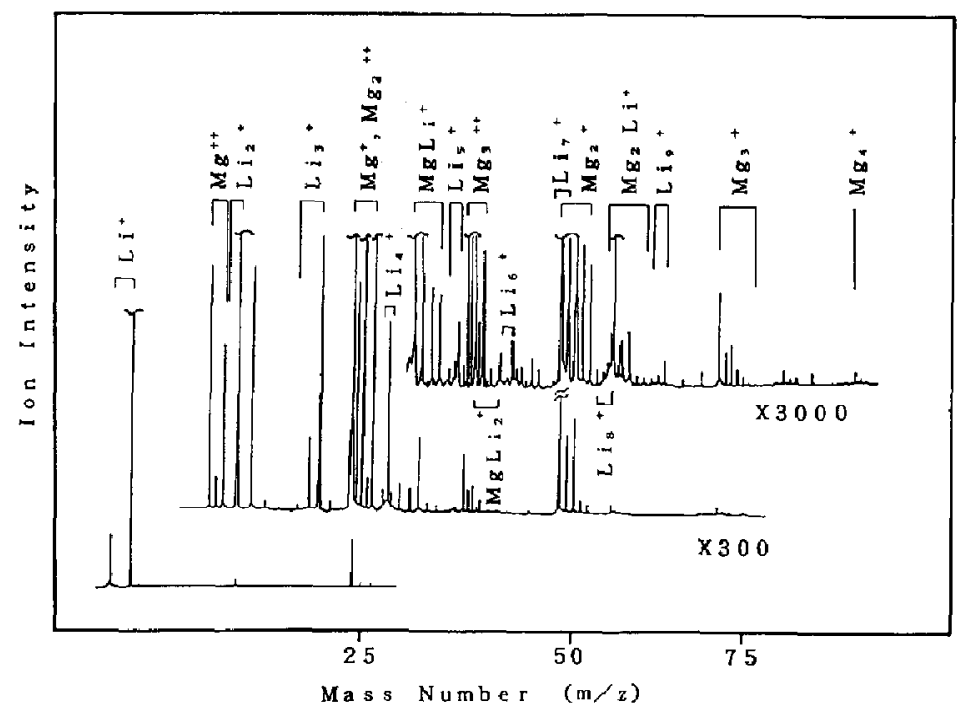

Figure 1. Mass spectrum of ions ejected from the Li-Mg alloy LMIS. Acceleration voltage of ions is $7.7 \mathrm{kV}$, and the total ion current $15 \mu \mathrm{A}$.

\section{Experimental}

We used a needle-type LMIS. The ion source consists of a needle emitter, a reservoir for liquid metal, a heater to melt source material, and an extractor electrode. Because the detailed structure of the LMIS has been described elsewhere $[7]$ it is only briefly mentioned here. This source has the following characteristics: (1) Source material is enclosed within a capped reservoir, (2) the reservoir and the needle tip are heated by electron bombardment, and (3) the needle can be moved back and forth from outside of the vacuum. Because of the closed-type reservoir, waste of source material through thermal evaporation is suppressed. Baking the emitter tip by electron bombardment in cooperation with the mechanical movement of the tip facilitates wetting of the tip with liquid metal.

A mixture of small pieces (ca. $1 \mathrm{~mm}^{3}$ cubes) of Li and $\mathrm{Mg}$ metals was used as source material. The purities of the metals are $99.9 \%$ for $\mathrm{Li}$ and $99.99 \%$ for $\mathrm{Mg}$. To avoid chemical reactions of $\mathrm{Li}$ metal with water vapor and oxygen in ambient gas, loading of the metals was carried out in a glove box filled with Ar gas. The amount of each metal loaded in the reservoir was adjusted to make an alloy with composition of approximately 10 atom \% Mg. An iron or tungsten wire with a diameter of $0.5 \mathrm{~mm}$ and sharpened to a tip radius of several micrometers by conventional electrochemical etching was used as a needle emitter. The mobile function of the needle facilitates mixing the molten pieces of the metals, making the liquid alloy uniform.

The needle tip covered with a liquid film is subjected to a high electric freld by applying a high positive voltage, typically $4-10 \mathrm{kV}$, to the needle against the extractor electrode (grounded). The mass distribution of ions ejected from the tip was measured with a single-focusing magnetic analyzer with an orbital radius of $20 \mathrm{~cm}$.

\section{Results and Discussion}

Figure 1 shows a mass spectrum of ions ejected from the $\mathrm{Li}-\mathrm{Mg}(10$ atom \%) alloy LMIS at a total emission current $I_{t}=15 \mu \mathrm{A}$. The presence of isotopes of $\mathrm{Li}$ (natural abundance of ${ }^{6} \mathrm{Li}$ is $7.5 \% ;{ }^{7} \mathrm{Li}, 92.5 \%$ ) and $\mathrm{Mg}$ $\left({ }^{24} \mathrm{Mg}, 78.7 \%,{ }^{25} \mathrm{Mg}, 10.1 \%,{ }^{26} \mathrm{Mg}, 11.2 \%\right)$ makes it difficult to identify peaks at high mass. For each cluster ion a mass pattern due to the isotopic combination is calculated by using the natural abundances of the isotopes. When different ion species pile up in a peak, the relative contribution of each species to the peak is estimated by comparing the calculated intensity distribution with the observed one.

For homonuclear clusters, $\mathrm{Li}_{n}^{+}$up to $n=9$ and $\mathrm{Mg}_{n}^{+}$up to $n=4$ are observed. The relative intensities of $\mathrm{Li}_{n}^{+}$and $\mathrm{Mg}_{n}^{+}$as a function of $n$ are plotted in a logarithmic scale as shown in Figure 2 together with that of $\mathrm{Li}_{n}^{+}$ejected from a pure Li LMIS. The $\mathrm{Li}_{n}^{+}$ abundance spectrum from the pure Li LMIS shows pronounced features; intensity drops after $n=3$ and 7. These structures in the spectra are attributed to the size-dependent stability of ionized clusters against fragmentation $[8,9]$; that is, a cluster at a higher step edge of intensity variation has higher stability than the neighboring clusters of the same size. However, the $\mathrm{Li}_{n}^{+}$abundance obtained from the alloy LMIS decreases with the cluster size more rapidly than that from the pure LMIS, and thus the abundance spectrum shows less pronounced structures (steps at $n=3$ and 7). The formation of $\mathrm{Li}_{n}^{+}$clusters seems to be suppressed by the existence of another element, $\mathrm{Mg}$, in the liquid metal.

The $\mathrm{Mg}_{n}^{+}$abundance curve also shows a steeply decreasing intensity with the size, and no structure is 


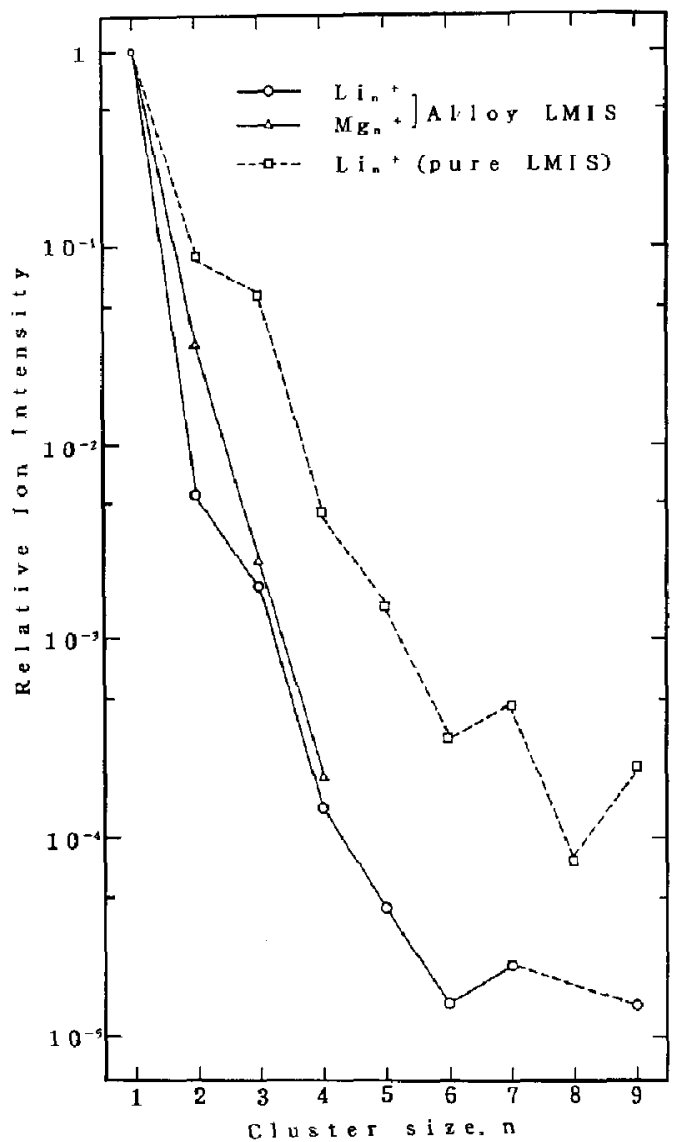

Figure 2. Relative ion intensity of $\mathrm{Li}_{n}^{+}(O)$ and $\mathrm{Mg}_{n}^{+}(\triangle)$ from the alloy. Intensity of $\mathrm{Li}_{t}^{+}$from a pure Li LMIS is also plotted (). Ion intensity is normalized to the respective monomer intensity. For the alloy source the intensity of $\mathrm{Li}_{B}^{+}$cannot be measured because the intensity of this cluster is too weak to separate peaks of this cluster from those of $\mathrm{Mg}_{2} \mathrm{Li}^{+}$. Therefore, the intensities of $\mathrm{Li}_{7}^{+}$and $\mathrm{Lig}_{9}^{+}$are connected by a dashed line.

found. This monotonic change of the intensity is consistent with the monotonic variation in cohesive energy of $\mathrm{Mg}_{n}^{+}$as a function of $n$ calculated by Durand [10]. The cohesive energy per atom calculated up to $n=7$ decreases monotonically.

Heteronuclear clusters detected are $\mathrm{MgLi}^{+}, \mathrm{MgLi}_{2}^{+}$, and $\mathrm{Mg}_{2} \mathrm{Li}^{+}$. Among them $\mathrm{MgLi}^{+}$is dominant. A theoretical study [11] on geometrical and electronic structures of $\mathrm{MgLi}_{k}^{+}$shows an odd-even alternation in the binding energy variation as a function of $k$; cationic clusters with odd $k$ (total number of valence electrons is even) have higher binding energies than the neighboring cationic clusters with even $k$. Because in the present experiment only the first two clusters in the $\mathrm{MgLi}_{k}^{+}$series are detected, no comparison with the theoretical study on the cluster stability can be made.

Figure 3 shows a magnified spectrum in a mass range around $\mathrm{Mg}^{+}$. Peaks with half-integer mass-to-

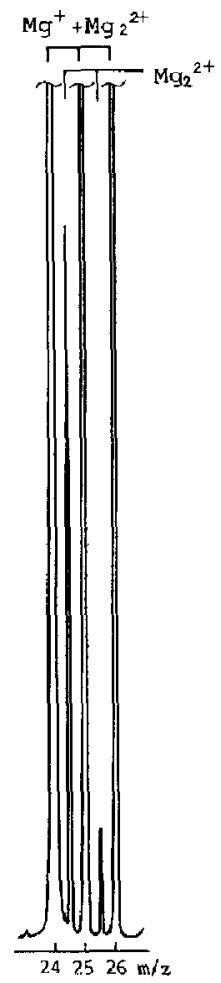

Figure 3. Magnified mass spectrum in a mass range between $\mathrm{m} / \mathrm{z} 24$ and 26 (corresponding to $\mathrm{Mg}^{+}$). Peaks at $\mathrm{m} / \mathrm{z} 24.5$ and 25.5 are due to doubly charged $\mathrm{Mg}$ dimers.

charge ratios 24.5 and 25.5 are observed, indicating the presence of doubly charged $\mathrm{Mg}$ dimers. The observed mass pattern between $m / z 24$ and 26 cannot be explained by other doubly charged clusters, including heteronuclear clusters $\mathrm{Mg}_{m} \mathrm{Li}_{n}^{2+}$. A mass pattern composed of $\mathrm{Mg}^{+}$and $\mathrm{Mg}_{2}^{2+}$ is calculated and is compared with the observed one in Figure 4 . The intensity is shown on a logarithmic scale. In the calculation, the relative height of isotope peaks is assumed to reflect the natural abundance of the isotopes. The abundance ratio of $\mathrm{Mg}^{+}$and $\mathrm{Mg}_{2}^{2+}$ is adjusted to accurately reproduce the observed pattern. The shaded parts in Figure $4 \mathrm{~b}$ show the contribution from the doubly charged dimer (the abundance ratio of ${ }^{24} \mathrm{Mg}_{2}^{2+}{ }^{24} \mathrm{Mg}^{+}$at $m / z 24$ is taken as 0.06 ). The agreement between the observed and calculated patterns leads to the conclusion that the mass peaks between 24 and $26 \mathrm{u}$ are from $\mathrm{Mg}^{+}$and $\mathrm{Mg}_{2}^{2+}$.

Concerning other multiply charged clusters, $\mathrm{Mg}_{3}^{2+}$ is also observed. The observation of doubly charged diatomic and triatomic clusters is surprising, because the Coulomb repulsion between the two holes should compel such small clusters to explode into two singly charged fragments.

The free atoms of divalent elements have an $s^{2}$ closed-shell atomic configuration. The atoms may be considered to keep essentially this ground-state structure in small clusters. On the other hand, bulk Mg 


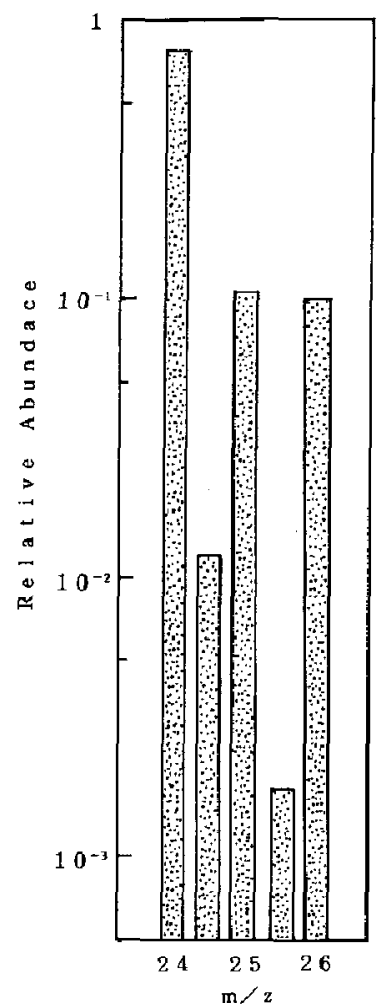

A

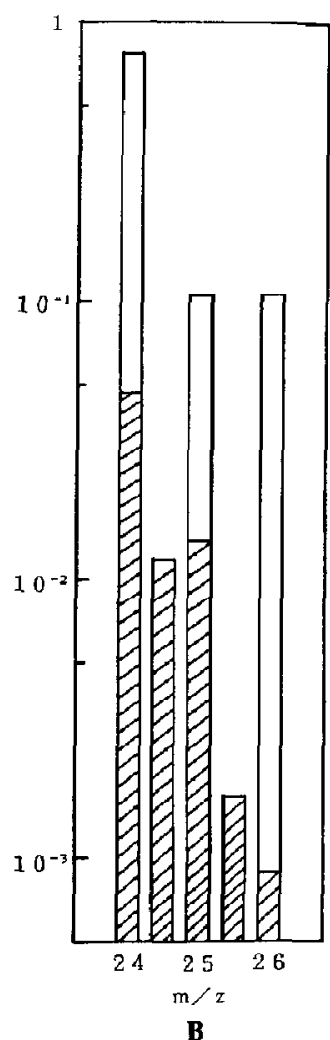

B
Figure 4. (a) Observed mass pattern between $m / z 24$ and 26; (b) calculated mass pattern composed of $\mathrm{Mg}^{+}$and $\mathrm{Mg}_{2}^{2+}$ with the intensity ratio ${ }^{24} \mathrm{Mg}_{2}^{2+} /{ }^{24} \mathrm{Mg}^{+}$of 0.06 . Shaded parts in (b) show contribution from $\mathrm{Mg}_{2}^{2+}$.

has metallic character that is due to the overlap between the filled $s$ and empty $p$ bands. Therefore, the Mg clusters smaller than a certain size have van der Waals bonding, and larger clusters transform to metallic bonding. The dissociation energy $D_{0}$ of an $\mathrm{Mg}$ dimer in the ground state is only $0.050 \mathrm{eV}$ [12]. The value of the binding energy per atom for the bulk, $E_{\mathrm{B}}$, is $1.53 \mathrm{eV}$ [13].

The stabilities of doubly charged clusters are evaluated by using the liquid-drop model and the chainlike model. The first model, which assumes that the two charges are located on opposite sides of a spherical droplet of $n$ atoms, yields a repulsive Coulomb energy $E_{C}=e^{2} / 2 r_{a} n^{1 / 3}$, where $e$ is an elementary electric charge and $r_{\mathrm{a}}$ is the atomic radius in a droplet. The value of $r_{a}$ lies somewhere between the bulk atomic radius $\left(2 r_{a}=3.54 \AA\right)$ and the equilibrium bond length for $\mathrm{Mg}_{2}\left(2 r_{\mathrm{a}}=3.89 \AA\right)$. According to the spherical liquid-drop model with a bare Coulomb interaction (no screening) and the bulk binding energy, doubly charged clusters are stable above a threshold value $n_{\mathrm{s}}^{*}$ obtained from $E_{\mathrm{C}}=E_{\mathrm{B}}$ [4]. This leads to a critical value $12<n_{s}^{*}<16$, which is inconsistent with our observations. When the binding energy of the $\mathrm{Mg}$ dimer is used as $E_{\mathrm{B}}, n_{\mathrm{s}}^{*}$ will abviously result in a value that is too high. In the second model, which assumes a linear configuration with the two charges localized at the outermost atoms, an empirical rough criterion of $E_{\mathrm{C}}<1.4 E_{\mathrm{B}}$ for a stable doubly charged cluster is obtained. According to this model, the critical number $n_{\mathrm{c}}^{*}$ is given by $E_{\mathrm{C}}=e^{2} / 2 r_{\mathrm{a}}\left(n_{\mathrm{c}}^{*}-1\right)=1.4 E_{\mathrm{B}}$ [5]. With $2 r_{\mathrm{a}}=3.54 \AA$ or $3.89 \AA$ and $E_{\mathrm{B}}=1.53 \mathrm{eV}$ as the binding energy of the bulk, $n_{c}^{*}=3$ is obtained. Although this critical size apparently agrees with the observed one, this calculated result loses self-consistency; in this small size range, the assumption of bulk binding energy is not valid. When the dimer binding energy is used, $n_{c}^{*}$ will again result in a value that is too high $\left(n_{c}^{*}=60\right)$. In conclusion, none of these models explains our experimental observations.

The discrepancy may be due to the neglect of polarization forces. Although $\mathrm{Mg}$ atoms in a neutral cluster are held together by weak dispersion forces, in a positively ionized $\mathrm{Mg}$ cluster polarization forces between an ion and atoms bind them tightly. The polarization forces are the main stabilizing factors when the charges are localized on a single atom. However, the true eigenfunction will probably be a mixing of two configurations, two charges on a single atom and charges on two different atoms. The stability of doubly charged $\mathrm{Mg}$ dimer and trimer may also be explained from the point of view of molecular orbital theory. Whether the charges are on a single atom or two atoms, the two $s$ valence electrons of $\mathrm{Mg}_{2}^{2+}$ will find the os bonding molecular orbital and leave the ( $\sigma s)^{*}$ antibonding orbital empty. This strong bonding should create a local minimum on the otherwise repulsive potential energy surface. Similar argu* ments apply to $\mathrm{Mg}_{3}^{2+}$.

Using a model Hamiltonian including the polarization energy and the interactions between induced dipoles, Durand et al. [14] calculated potential energy surfaces of $\mathrm{Mg}_{n}^{z+}(n \leq 5 ; z=1,2)$ and showed that both $\mathrm{Mg}_{2}^{2+}$ and $\mathrm{Mg}_{3}^{2+}$ have local minima in the potential energy surfaces.

Tsong [15] observed doubly charged molybdenum dimer in pulsed-laser stimulated field evaporation, which is the only observation of doubly charged diatomic cluster ions of a metal. As far as divalent (group II) elements are concerned, $\mathrm{Mg}_{2}^{2+}$ presently observed is the smallest doubly charged cluster. $\mathrm{Mg}_{3}^{2+}$ is the next smallest doubly charged cluster. For $\mathrm{Hg}$, isoelectronic with $\mathrm{Mg}, \mathrm{Hg}_{5}^{2+}$ is known to be the smallest doubly charged cluster observed experimentally [16], though the existence of a potential barrier against fragmentation for $\mathrm{Hg}_{2}^{2+}$ has been found theoretically [17]. The $\mathrm{Hg}$ cluster ions were produced by photoionization of neutral clusters formed through free jet expansion. The method of generation of cluster ions affects the observability of metastable multiply charged clusters [14, 18], as will be discussed below for $\mathrm{Mg}$.

The existence of local minima in the potential energy surface of $\mathrm{Mg}_{n}^{2+}$ is not sufficient to ensure the observability of these clusters, because these minima 
are significantly higher than the potential energy of $\mathrm{Mg}_{p}^{+}+\mathrm{Mg}_{q}^{+}(p+q=n)$ separated to an infinite distance from each other. Clusters might tunnel through the barrier, or, if excited to a state higher in energy than the barrier height, they will break into fragment ions. Another factor that prevents the observation of doubly charged clusters is the great difference between the geometry of the neutral $\mathrm{Mg}_{n}$ and that of $\mathrm{Mg}_{n}^{2+}$ in the local minimum [14]. A vertical double ionization of the neutral species would not lead the stable doubly charged cluster, because it forms the cluster in a region outside the energy barrier.

Durand et al. [14] pointed out that the stable geometries of $\mathrm{Mg}_{n}^{+}$and $\mathrm{Mg}_{n}^{2+}$ are quite similar. This means that an ionization from $\mathrm{Mg}_{n}^{+}$to $\mathrm{Mg}_{n}^{2+}$ would lead to a structure close to the bottom of the $\mathrm{Mg}_{n}^{2+}$ local minimum, providing an opportunity for this structure to have a significant lifetime. This theoretical finding indicates that the experimentally observed $\mathrm{Mg}_{2}^{2+}$ and $\mathrm{Mg}_{3}^{2+}$ are not produced by the simultaneous double ionization of free neutral $\mathrm{Mg}_{2}$ and $\mathrm{Mg}_{3}$, but rather are formed by ionization of $\mathrm{Mg}_{2}^{+}$and $\mathrm{Mg}_{3}^{+}$, respectively, field-evaporated (as ions) from the liquid surface. The latter process is known as postionization [6]. This process successfully explains the emission of doubly charged monomers from the LMIS for various metals. The present result suggests that postionization occurs for clusters as well as monomers.

The total flight time of $\mathrm{Mg}_{2}^{2+}$ from formation to detection is about $2 \mu \mathrm{s}$. We have not measured the intensity change of $\mathrm{Mg}_{2}^{2+}$ versus ion acceleration voltage (flight time), so the lifetime of $\mathrm{Mg}_{2}^{2+}$ cannot be deduced. However, considering the relatively high intensity of $\mathrm{Mg}_{2}^{2+}$ (6\% of the intensity of $\mathrm{Mg}^{+}$, and two to three times $\mathrm{Mg}_{2}^{+}$intensity), the lifetime may be on the order of microseconds or more.

\section{References}

1. See, for example, (a) Proceedings of the 4th International Symposium on Small Particles and Inorganic Clusters ( $Z$. Phys. $D$ 1989, 12, nos. 1-4): (b) Sugano, S., et al., Eds. Microclusters; Springer-Verlag: Berlin, 1987.

2. Sattler, K.; Mühlbach, J.; Echt, O.; Pfau, P.; Recknagel, E. Phys. Rev. Lett. 1981, 47, 160-163.

3. Echt, O. In Physics and Chemistry of Small Clusters; Jena, P. et al., Eds.; Plenumt: New York, 1987; p. 623.

4. Tománek, D.; Mukherjee, S,; Bennemann, K. H. Phys. Rev. B 1983, 28, 665-673.

5. Drachsel, W.; Jentsch, Th.; Gingerich, K. A; Block, J. H. Surface Sci. 1985, 156, 173-182.

6. Kingham, D. R. Surface Sci, 1982, 116, 273-301.

7. Noda, T.; Okutani, T.; Yagi, K.; Tamura, H.; Okano, H.; Watanabe, H. Int. I. Mass Spectrom. Ion Phys. 1983, 46, 15- 18.

8. Joyes, P.; Sudraud, P. Surface Sci. 1985, 156, 451-458.

9. Saito, Y.; Noda, T. Z. Phys. D 1989, 12, 127-129.

10. Durand, G. J. Chem. Phys. 1989, 91, 6225-6237.

11. Fantucci, P.; Koutecký, V. B.; Pewestorf, W.; Koutecký, J. J. Chem. Phys, 1989, 91, 4229-4241.

12. Huber, K. P.; Herzberg, G. Constants of Diatomic Molecules; Van Nostrand: New York, 1989; p. 394.

13. Kittel, C. Introduction to Solid State Physics, 4th ed.; Wiley: New York, 1971; p. 96.

14. Durand, G.; Daudey, J.-P;; Malrieu, J.-P. I. Phys. (Paris) 1986, 47, 1335- 1346.

15. 'Tsong, 'I'. T. J. Chem. Phys. 1986, 85, 639-640.

16. Bréchignac, C.; Broyer, M.; Cahuzac, Ph.; Delacretaz, G.; Labastic, P.; Wöste, L. Chem. Phys. Lett. 1985, 118, 174-178.

17. Neisler, R. P.; Pitzer, K. S. I. Phys. Chem. 1987, 91 1084-1087.

18. Khanna, S. N.; Reuse, F.; Buttet, J. Phys. Reo. Lett. 1988, $61,535-538$. 